\title{
Getting instructions from mum
}

histone
H3 Lys27
trimethylation
(H3K27me3)
is important
for trans-
generational
epigenetic
inheritance

In gametes, most of the epigenetic modifications are erased before fertilization, but some epigenetic traits are retained and can be passed to the progeny in a process that remains poorly understood. Two studies now show that histone H3 Lys27 trimethylation (H3K27me3) is important for transgenerational epigenetic inheritance.

$\mathrm{H} 3 \mathrm{~K} 27 \mathrm{me} 3$ is associated with chromatin compaction and transcriptional silencing. Zenk et al. found considerable levels of $\mathrm{H} 3 \mathrm{~K} 27 \mathrm{me} 3$ in Drosophila melanogaster oocytes and early embryos. H3K27me3 was still detected in the fly zygotes at fertilization when enhancer of zeste $(\mathrm{E}(\mathrm{z}))$ - which catalyses H3K27me3 - was depleted during late oogenesis, but was lost in embryos after two to three nuclear divisions, which indicated that this epigenetic mark is inherited from the maternal germ line. The loss of $\mathrm{H} 3 \mathrm{~K} 27 \mathrm{me} 3$ was associated with embryonic lethality, which could not be rescued by embryonic expression of $\mathrm{E}(\mathrm{z})$ and re-establishment of H3K27me3. Thus, $\mathrm{H} 3 \mathrm{~K} 27 \mathrm{me} 3$ is maternally deposited in zygotes and further maintained in

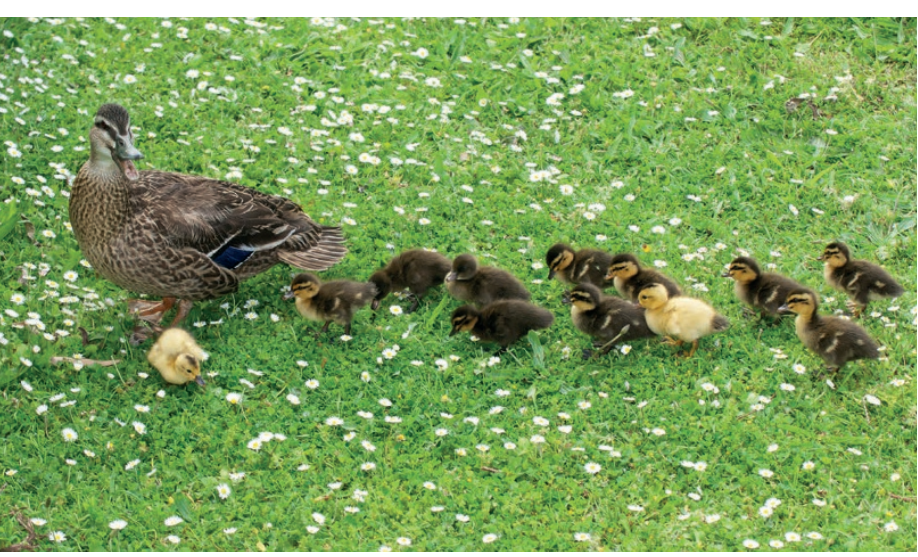

Getty/Byronsdad embryos by maternal $\mathrm{E}(\mathrm{z})$, and this maternal inheritance is crucial for embryonic development.

In embryos that lacked maternal $\mathrm{E}(\mathrm{z}), \mathrm{H} 3 \mathrm{~K} 27 \mathrm{me} 3$ was replaced by H3K27 acetylation (H3K27ac). This alteration from repressive to activating histone modification could have profound consequences for embryonic gene expression, in particular on its onset, which occurs during zygotic genome activation (ZGA). Indeed, following $\mathrm{E}(\mathrm{z})$ depletion, the expression of genes now aberrantly marked by H3K27ac - many of which were found to be regulators of developmental programmes - was upregulated at ZGA. This indicated that in the fly the loss of maternal H3K27me3 inheritance leads to precocious activation of a subset of genes, which interferes with normal developmental programmes.

Inoue et al. studied epigenetic inheritance in the mouse and identified multiple parental-specific DNase I hypersensitive sites (DHSs; which mark open chromatin) in the mouse zygote and morula embryos. Such differences in chromatin accessibility between the paternal and maternal genomes were revealed to be associated with imprinted gene expression at ZGA and in morula embryos.

In contrast to maternal DHSs, which were detected already in the oocyte, most paternal DHSs were not detected in the sperm. This suggested that sperm chromatin is remodelled after fertilization, whereas maternal chromatin resists such remodelling. To understand how maternal DNA is silenced before fertilization to allow paternal gene expression in the embryo, the authors investigated patterns of DNA methylation - the only established mammalian imprinting mark. Surprisingly, maternal DNA corresponding to paternal DHSs was mostly hypomethylated. Instead, these regions were reproducibly marked by H3K27me3, and on overexpression of the H3K27me3-specific demethylase KDM6B (also known as JMJD3), many paternal-specific DHSs were now also present in the maternal DNA, concurrent with their activation (resulting in bi-allelic expression).

The authors identified 76 genes associated with paternal DHSs that were marked by H3K27me3 in the maternal genome. Among those, 27 genes exhibited biased paternal expression in morula embryos, which was turned into bi-allelic expression in the presence of ectopic KDM6B. This collectively indicated that $\mathrm{H} 3 \mathrm{~K} 27 \mathrm{me} 3$ is a previously unrecognized mechanism of genomic imprinting in mammals, which is responsible for chromatin compaction and silencing of maternal alleles of a subset of genes.

In summary, maternal inheritance of $\mathrm{H} 3 \mathrm{~K} 27 \mathrm{me} 3$ has emerged as an evolutionarily conserved mechanism that regulates gene expression during early animal development. In the future, it will be interesting to study how specific loci are imprinted by H3K27me3 and how they escape erasure during gamete epigenetic reprogramming.

Paulina Strzyz

ORIGINAL ARTICLES Zenk, F. et al. Germ lineinherited $\mathrm{H} 3 \mathrm{~K} 27 \mathrm{me} 3$ restricts enhancer function during maternal-to-zygotic transition. Science 357, 212-216 (2017) | Inoue, A. et al. Maternal H3K27me 3 controls DNA methylationindependent imprinting. Nature http://dx.doi. org/10.1038/nature23262 (2017) 